\title{
Impacto del DR-CAFTA en las exportaciones del sector agrícola y su contribución al crecimiento económico de Honduras, periodo 2000-2011
}

\author{
José S. Menjívar' \\ José Luis Martínez
}

\section{RESUMEN}

El desarrollo de la política comercial en las economías pequeñas y en vías de desarrollo juega un rol importante en el dinamismo de la actividad económica. A raíz de ello, esta investigación se centra en analizar el impacto generado por la entrada en vigencia del DR-CAFTA en las exportaciones del sector agrícola y su contribución al crecimiento económico de Honduras. En este sentido, dadas las incertidumbres sobre el impacto del DR-CAFTA en la economía hondureña, esta investigación se realizó basándose en el enfoque cuantitativo de carácter explicativo y retrospectivo en el marco de las ganancias o pérdidas del mismo. Asimismo, se fundamentó en fuentes secundarias, utilizando como principal técnica de análisis la estadística inferencial a través de la aplicación de modelos de regresión lineal múltiple de mínimos cuadrados ordinarios (MCO) en el software estadístico denominado STATA (por sus siglas en inglés).

De acuerdo a los diferentes escenarios realizados econométricamente, se encontró que la entrada en vigencia del DR-CAFTA ha generado un impacto positivo en las exportaciones del sector agrícola, el mayor impacto del acuerdo recae en las exportaciones agrícolas hacia República Dominicana, en donde ha contribuido en un $80.8 \%$ en el crecimiento anual de las exportaciones, comparado con un escenario sin la apertura del acuerdo; en un segundo lugar se encuentra Guatemala con una contribución del $78.4 \%$, en un tercer lugar Costa Rica con $62.9 \%$, cuarto lugar Nicaragua con $51.1 \%$, quinto lugar El Salvador con $44.3 \%$ y en último lugar EE.UU. con $43.5 \%$.

Palabras clave: DR-CAFTA, crecimiento económico, exportaciones sector agrícola.

\footnotetext{
${ }^{1}$ Beneficiario de una beca básica de la DICYP. Estudiante de la Maestría en Metodologías de la Investigación Económica y Social, Facultad de Ciencias Económicas, UNAH: jose.menjivar@unah.edu.hn

${ }^{2}$ Asesor, Departamento de Economía, Facultad de Ciencias Económicas de la UNAH: mjoseluis321@yahoo.com
} 


\section{ABSTRACT}

dynamism of the economic activity. As a result of this, the present investigation focuses in analyzing the impact generated with the come into force of the DR-CAFTA in the exportations of the agricultural sector and its contribution in the economic growth of Honduras. In this sense, given the uncertainties about the impact of DRCAFTA in the Honduran economy, this research was conducted based on the quantitative approach and retrospective explanatory under gains or losses thereof. Also, it was based on secondary sources, using as main analysis technique the inferential statistics, through the application of multiple linear regression of Ordinary Least Squares model (OLS) in the statistical software STATA called by its acronym in English.

According to the different scenarios, econometrically speaking, it was found that the entry into force of DR-CAFTA has had a positive impact on exports of the agricultural sector, the greatest impact of the agreement lies in agricultural exports was to the Dominican Republic where it has contributed $80.8 \%$ annual growth in exports, compared to a scenario without opening the agreement, in A second place is found Guatemala with a contribution of $78.4 \%$, in third place with $62.9 \%$ Costa Rica, Nicaragua fourth with $51.1 \%$ El Salvador in the fifth place with $44.3 \%$ and in the last place USA with $43.5 \%$.

Keywords: DR-CAFTA, economic growth, exports agricultural sector.

\section{INTRODUCCIÓN}

Según la Secretaría de Industria y Comercio, el uno de abril de 2006 entró en vigencia el tratado de libre comercio, DR-CAFTA, entre los Estados Unidos de América (EE.UU.), Centroamérica y República Dominicana; aunque las negociaciones del tratado culminaron en agosto de 2004, la entrada en vigencia para los países miembros se dio varios años después.

Para entender de forma efectiva las contribuciones e implicaciones de la apertura comercial, en este caso el DR-CAFTA, es necesario analizar una serie de indicadores, analizando el déficit o superávit que se tiene entre las economías participantes antes y después de haber firmado el tratado comercial (USAID, 2011). En este sentido, el desarrollo de esta investigación se fundamentó en brindar un aporte al estudio de la economía internacional de Honduras en materia agrícola, brindando nuevos aspectos a considerar por los negociadores al momento de la firma de nuevos tratados y en la administración adecuada de los ya existentes, 
puesto que los recientes estudios enfocados a la apertura comercial describen con poca profundidad el impacto del DR-CAFTA en el sector agrícola y en las potenciales condiciones de competitividad en las exportaciones de los principales productos agrícolas, sumado a la sensible variación que muestra la oferta agrícola en materia de exportación durante la última década.

Los resultados provenientes de esta investigación están encaminados en contribuir al desarrollo de la investigación académica enmarcados en herramientas estadísticas utilizadas principalmente por los economistas y profesionales de las ciencias sociales. Asimismo, tiene una fuerte aplicación para la toma de decisiones de las instituciones del sector público y privado de la economía hondureña.

En el marco del comercio internacional analizar el impacto de un tratado de libre comercio implica la utilización de herramientas cuantitativas tales como modelos de equilibrio parcial y en su mayor parte de orientación econométrica. En este sentido, para efectos de esta investigación el análisis explicativo se fundamentó en el modelo gravitacional propuesto por Tinbergen en 1962, el cual pone en juego la fuerza gravitacional de la ley de Newton, este modelo explica el crecimiento de las exportaciones con relación al tamaño económico (en términos del PIB) de las economías participantes y su distancia (USAID, 2011).

Según la investigación Determinantes de las exportaciones mundiales de manufacturas a China 1990-2006 (Álvarez y Figueroa, etal, 2009), se considera que una de las metodologías más utilizadas y exitosas para estudiar los factores que determinan el intercambio de bienes y su importancia cuantitativa es el denominado modelo de gravedad propuesto inicialmente por Tinbergen (1962). Este modelo ha tenido diversas aplicaciones, tales como en La evaluación del impacto del DRCAFTA en los sectores productivos de la República Dominicana (USAID, 2011), MERCOSUR, tendencias de internacionalización y capacidades tecnológicas (Álvarez y Fischer, et al, 2013), el MERCOSUR como plataforma de exportación para la industria automotriz (Arza, 2011) y México: dinámica de las exportaciones manufactureras (Cuevas, 2010).

De acuerdo con Álvarez (2009), la especificación estándar de la aplicación de modelos gravitacionales en el análisis del comercio internacional impone una simetría que no concuerda con los datos, lo cual conduce a estimaciones sesgadas. Muchos investigadores opinan que este tipo de ecuaciones se deberían trabajar con datos de panel, debido a que las estimaciones por mínimos cuadrados ordinarios (MCO) no reflejan la heterogeneidad inherente a las corrientes comerciales bilaterales. 
Para efectos de lograr obtener el impacto del DR-CAFTA en las exportaciones del sector agrícola de la economía hondureña, las ecuaciones se estiman desde el punto de vista de $\mathrm{MCO}$, producto de la falta de información coherente para trabajar con datos de panel. Igualmente, se toma como base para realizar las especificaciones econométricas el estudio de USAID en el año 2011, vinculado a obtener la evaluación del impacto del DR-CAFTA en los sectores productivos de la República Dominicana.

\section{METODOLOGÍA}

En el proceso de evaluación del impacto del DR-CAFTA en las exportaciones del sector agrícola y su aporte al crecimiento económico de Honduras, metodológicamente se limitó a realizar un análisis retrospectivo que involucrara únicamente la ejecución del tratado, acompañado de un análisis multivariado después de su entrada en vigencia, enfocándose en los efectos de carácter macroeconómico como resultado del intercambio comercial entre las economías que lo integran.

Para el caso del análisis retrospectivo se utilizó un enfoque basado en indicadores, centrándose en analizar los efectos en los mercados miembros, destacándose que solo se necesitaron datos de flujos de comercio y valores para algunos parámetros de comportamiento. Por otra parte, el análisis multivariado tuvo una mayor orientación econométrica, considerando las principales subvariables que implica el tratado comercial en lo referente al sector agrícola y al crecimiento económico de Honduras.

Desde el punto de vista del alcance de la investigación, dada la incertidumbre sobre los efectos de los tratados de libre comercio, se realizó un enfoque de investigación cuantitativo deductivo de carácter explicativo y retrospectivo, en el marco de las ganancias o pérdidas del mismo, con el fin de evaluar el impacto en el volumen de comercio del sector agrícola antes y después de la entrada en vigencia del acuerdo. En este sentido, en la determinación del impacto de un tratado de libre comercio, tal es el caso del DR-CAFTA, se presenta una serie de complejidades de orden cuantitativo, es por ello que se tomó como periodo de estudio el flujo comercial de los últimos 12 años, considerando como variable las exportaciones del sector agrícola hacia EE.UU., Centroamérica y República Dominicana. También se consideró el crecimiento económico medido a través del PIB como una segunda variable en el mismo periodo de tiempo para la economía hondureña.

El instrumento o técnica de recolección de datos que se llevó a cabo en el desarrollo de la investigación consistió en la recopilación y análisis de datos secundarios a 
través de fichas de registro, fundamentado en información disponible por los entes encargados a nivel nacional e internacional. La aplicación de este tipo de técnica se realizó con el propósito de obtener una base de datos con alto grado de confiabilidad en el proceso de tratamiento de la información. El proceso mediante el cual se desarrolló la recolección de datos cuantitativos consistió en:

1. Recopilación de datos secundarios durante el periodo 1990-2011 para las exportaciones totales en el marco del DR-CAFTA, tanto agrícolas como del resto de exportaciones, términos de intercambio, índice de producción agrícola, índice de volumen de exportación, tasa de inflación y el ingreso medido en términos del PIB; para el resto de indicadores se recopiló con base al periodo 2000-2011.

2. Creación de una base de datos en Microsoft Excel y STATA.

3. Codificación y estructuración de datos.

4. Recolección y análisis de datos, flexibles y oportunos.

5. Formular los datos cronológicamente.

6. Contrastar los datos de las fuentes nacionales con las de carácter internacional.

Una vez identificadas las fuentes de información y el diseño de las formas de recolección de datos cuantitativos, se procedió al tratamiento y análisis de datos a través de los paquetes estadísticos de Microsoft Excel y STATA, en donde se realizó la elaboración de cuadros, tablas de datos y modelos de inferencia estadística (regresión múltiple yANOVA).

Tomando en consideración el alcance de la investigación, la selección de las unidades de análisis fue de forma intencional o por conveniencia del investigador, quedando integrada de la siguiente manera:

1. Población: se determinó que la población a utilizar en la medición del impacto del DR-CAFTA en las exportaciones del sector agrícola serían las fuentes de datos oficiales de la economía hondureña y de organismos internacionales vinculadas a la obtención de indicadores de comercio internacional.

2. Unidades de información: correspondió a las entidades básicas mediante la cual se accedió a la unidad de análisis de la investigación, tales como: Banco Central de Honduras (BCH), Comisión Económica para los Pueblos de América Latina 
(CEPAL), sistema de bases de datos COMTRADE de las Naciones Unidas, sistema interactivo de gráficos de comercio internacional (SIGCI), Organización Mundial del Comercio (OMC), Sistema de Integración Económica Centroamericano (SIECA), Secretaría de Industria y Comercio (SIC), el Trade Performance Index (TPI), National Export Performance (NEP) y Magic Plus de la CEPAL.

\section{RESULTADOS}

Especificación econométrica del modelo de impacto delDR-CAFTA

Para efectos de esta investigación, el análisis explicativo se fundamentó en el modelo gravitacional propuesto por Tinbergen en 1962, el cual pone en juego la fuerza gravitacional de la ley de Newton. Este modelo explica el crecimiento de las exportaciones con relación al tamaño económico (en términos del PIB) de las economías participantes y su distancia:

\section{[1] FGij=(MiMj)/Dij}

Endonde:

FGij: fuerza gravitacional entre dos objetos i y j directamente proporcional a las masas de los objetos e indirectamente proporcional a la distancia entre ellos. MiMj: masas de los objetos.

Dij: distancia de los objetos.

Con el objeto de medir el impacto del DR-CAFTA en las exportaciones de Honduras es necesario convertir la ecuación [1] de modelo gravitacional en términos de un modelo econométrico a través de la aplicación de logaritmos, de donde se obtiene la ecuación [2]:

\section{[2] InFGij=InMi+InMj-InDij}

De la misma manera, esta ecuación [2] se puede expresar en función del crecimiento de las exportaciones de Honduras en el marco del DR-CAFTA hacia los EE.UU. y el resto de países que integran este acuerdo comercial. Por tanto, el tercer paso es adaptar la ecuación [1] en términos de las relaciones de las exportaciones, tal como se expresa en la ecuación [3], en donde se espera que exista una relación positiva entre los crecimientos de las exportaciones con el PIB del país importador y del país exportador, pero de manera negativa con la distancia entre los países: 
[3] Xijt=G((YiYj)/Dij)

Endonde:

G: Es una constante

Xijt: exportaciones de bienes y servicios del país i al país j

Yi:PIB del país exportador

Yj: PIB del país importador

Dij: Distancia entre el país i yj

La ecuación [3] está representada de forma lineal, pero si se le aplica logaritmos quedaría expresada de la siguiente manera [4]:

[4] $\ln X i j=B 0+B 1 \ln Y i t+B 2 \ln Y * j t+E i j t$

Endonde:

InXijt: exportaciones de bienes y servicios del país i al país j

InYit:PIB del país exportador en el año " $\mathrm{t}$ "

$\ln Y^{*} j t: P I B$ del país importador en el año " $t$ "

Eij: Errores residuales

En la búsqueda del impacto del DR-CAFTA es necesario tomar en cuenta las características de la economía hondureña que mayor incidencia pueden generar en el comportamiento de los niveles de exportación agrícola y en su totalidad, ante tal situación, la ecuación [4] para efectos de esta investigación queda determinada de la siguiente manera:

\section{[5] Xijt=B0+B1/nYijt+B2InTINit+B3InDRCAFTAt+B4InINDXit+InINFit+Eijt}

Endonde:

InYijt= In $Y^{*} j t-I n Y i j:$ el PIB del país importador se expresa en términos relativos al PIB del país exportador, siendo InYit: el ingreso para el país exportador y $\ln Y^{*} j t$ el ingreso para el país importador.

InTINit: el índice de relación términos de intercambio del país i en relación a las importaciones.

DRCAFTAt: es una variable dicotómica en donde se le asignan valores de "0" a los años antes de entrar en vigencia el DR-CAFTA y "1" para los años a partir de la entrada en vigencia del mismo.

INDXit: índice del volumen de las exportaciones tomando como base el año 2000.

INFit: los niveles de inflación por parte del país "i", en este caso la inflación de la economía hondureña. 
De acuerdo a las variables utilizadas en la cuantificación del impacto del DR-CAFTA en las exportaciones de la economía hondureña durante el periodo 2000-2011, los resultados esperados en sus respectivos coeficientes estarán integrados de la siguiente forma:

1. El coeficiente B1 correspondiente al ingreso del país importador en términos del ingreso del país exportador, se espera que muestre una relación positiva con respecto a la demanda externa de la economía hondureña.

2. El coeficiente B2 pertenece al índice de precios de intercambio, el cual se utiliza para medir la evolución relativa de los precios de las exportaciones y de las importaciones de la economía hondureña. Por tanto, se habla de deterioro de los precios de intercambio, el precio de los productos exportados tiende a disminuir comparado con el de los productos importados. Bajo este comportamiento se espera que los términos de intercambio muestren una relación positiva con los niveles de exportación.

3. El coeficiente B3 corresponde a la variable DR-CAFTA, en el cual se parte de que la entrada en vigencia del DR-CAFTA pretende ser el mecanismo que contribuye en un $75 \%$ al proceso de expansión de las exportaciones totales, dadas las limitaciones en recursos productivos e innovación del mercado. En este sentido, se espera una relación positiva respecto al crecimiento de las exportaciones.

4. El coeficiente B4 correspondiente al índice del volumen de las exportaciones; el cálculo de este índice se fundamenta en las exportaciones e importaciones clasificadas por producto, país de destino o procedencia, por tanto, se espera una relación positiva con respecto al crecimiento de las exportaciones.

5. El coeficiente B5 corresponde a la tasa de inflación de la economía hondureña, la cual pretende medir las variaciones generales de los precios.

Impacto del DR-CAFTA en las exportaciones totales del acuerdo por efecto de cada uno de los miembros del acuerdo

En lo que respecta al impacto del DR-CAFTA en las exportaciones totales de Honduras en el marco de este acuerdo, explicado por el efecto de la actividad económica de cada uno de los miembros del acuerdo, se encontró la estimación econométrica que se observa en la tabla 1. 
Tabla 1. Impacto del DR-CAFTA en las exportaciones totales del DR-CAFTA, 20002011
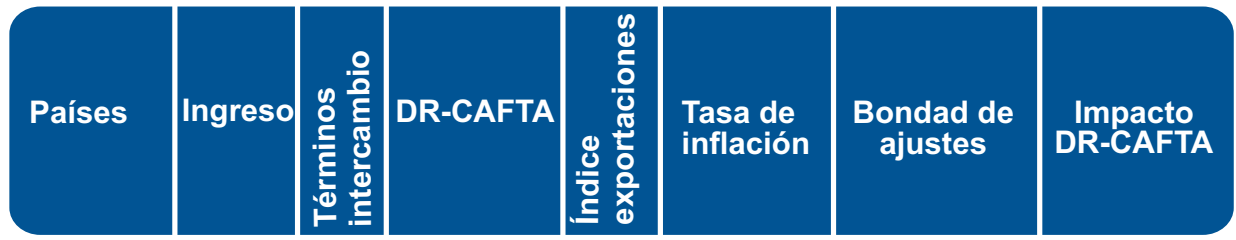

\begin{tabular}{l|l|l|}
\hline EE.UU. & -0.8 & 1.29 \\
\hline Guatemala & 0.44 & 1.16 \\
\hline El Salvador & 0.15 & 1.32 \\
\hline Costa Rica & 0.99 & 1.01 \\
\hline RD & 0.39 & 1.05 \\
\hline Nicaragua & 0.26 & 0.95 \\
\hline
\end{tabular}

\begin{tabular}{|c|c|}
\hline 0.39 & 0.6 \\
\hline 0.53 & 0.9 \\
\hline 0.59 & 0.8 \\
\hline 0.48 & 1.1 \\
\hline 0.5 & 0.9 \\
\hline 0.57 & \\
\hline
\end{tabular}

0.17

$91 \%$

$54.7 \%$

0.83

\begin{tabular}{l}
$-\quad 90 \%$ \\
\hline$-\quad 88 \%$
\end{tabular}

$63.0 \%$

$66.5 \%$

19

0.92

$-$

$92 \%$

$59.8 \%$

Fuente: elaboración propia con datos del BCH y Banco Mundial.

De acuerdo a los test individuales en el modelo especificado de la tabla 1, se parte de que los betas individuales y el modelo en su forma conjunta son estadísticamente significativos con un nivel de confianza del $95 \%$, manteniendo un nivel de confianza del $5 \%$. Por otra parte, los coeficientes de determinación o bondad del ajuste muestran que las variables independientes explican muy bien a los niveles de exportaciones totales de Honduras en el marco del DR-CAFTA.

En este sentido, las exportaciones totales hacia el DR-CAFTA están determinadas inversamente por el ingreso de los EE.UU. y de forma positiva a través del índice términos de intercambio, la entrada en vigencia del DR-CAFTA, índice de volumen de exportaciones y la tasa de inflación. En este sentido, la implementación de este acuerdo comercial sumado al ritmo de crecimiento en el ingreso de los EE.UU. en términos del ingreso de Honduras, ha contribuido en un $54.7 \%$ en el crecimiento anual de las exportaciones, comparado con un escenario sin la apertura del DRCAFTA.

El coeficiente estimado en este modelo para la variable DRCAFTA para EE.UU. es de 0.3975. Debido a que el modelo está en logaritmos, se debe tomar la inversa para determinar el impacto, por lo que estaría determinado de la siguiente forma:

DRCAFTA $=\left[\exp (0.3975-1)^{*} 100\right]$ DRCAFTA $=54.7 \%$

El impacto del DR-CAFTA influenciado por el comportamiento de la actividad económica de los EE.UU., se sustenta en que las exportaciones hacia este país son 
las que mayor prevalecen con una participación promedio del $63.4 \%$ (antes el $65 \%$ y después el $61.8 \%$ ) y un crecimiento del $9.6 \%$ (antes el $4.9 \%$ y después el $13.5 \%$ ). Asimismo, las exportaciones agrícolas crecieron a un ritmo del $8.8 \%$ durante el periodo 2006-2011; en contraste con una disminución del $17.5 \%$ en el 2009 y un 3.7 $\%$ en el 2006.

En lo que respecta a la balanza comercial agrícola, se mantiene un superávit de 4,185 millones de USD en las exportaciones de pescados y crustáceos, moluscos y demás invertebrados acuáticos (1,110.7 millones de USD); plantas vivas y productos de la floricultura (1,422.8 millones de USD); hortalizas, plantas, raíces y tubérculos alimenticios (217.5 millones de USD); frutas y frutos comestibles, cortezas de agrios (cítricos), melones o sandías (2,243.3 millones de USD); café, té, yerba mate y especias (612.5 millones de USD). En contraste con un déficit comercial agrícola de 1,936.5 millones de USD en el resto de las exportaciones agrícolas.

El impacto del DR-CAFTA influenciado por el comportamiento de la actividad económica de Guatemala se sustenta en que las exportaciones hacia este país se mantienen en un tercer lugar con respecto al total de este acuerdo, con una participación promedio del $11 \%$ (antes el $10.7 \%$ y después el $11.3 \%$ ), sumado a un crecimiento promedio del $13.1 \%$ (antes el $12.6 \%$ y después el $13.3 \%$ ). Igualmente, el impacto del DR-CAFTA influenciado por el comportamiento de la actividad económica de El Salvador se sustenta en que las exportaciones hacia este país se mantienen en un segundo lugar con respecto al total de este acuerdo, con una participación promedio del $15.1 \%$ (antes el $16.1 \%$ y después el $14 \%$ ), junto a un crecimiento del $10.2 \%$ (antes el $6.7 \%$ y después el $13.1 \%$ ).

Por otra parte, el impacto del DR-CAFTA influenciado por el comportamiento de la actividad económica de Costa Rica se sustenta en que las exportaciones hacia este país se mantienen en un quinto lugar con respecto al total de este acuerdo, con una participación promedio del $3.7 \%$ (antes el $3.3 \%$ y después el $4.1 \%$ ) y un crecimiento del $16.8 \%$ (antes una disminución del $3 \%$ y después un aumento del $33.4 \%$ ); y por último, República Dominicana con una participación del $0.9 \%$ (antes el $0.4 \%$ y después el $1.4 \%$ ). Asimismo, el impacto del DR-CAFTA influenciado por el comportamiento de la actividad económica de República Dominicana se sustenta en que las exportaciones hacia este país se mantienen en un sexto lugar con relación al total de este acuerdo, con una participación promedio del $0.9 \%$ (antes el $0.4 \%$ y después el $1.4 \%$ ). De igual forma, el impacto del DR-CAFTAinfluenciado por el comportamiento de la actividad económica de República Dominicana se sustenta en que las exportaciones hacia este país se mantienen en un cuarto lugar con una 
participación promedio del $5.9 \%$ (antes el $4.4 \%$ y después el $7.3 \%$ ) y un crecimiento del $23.2 \%$ (antes $27.8 \%$ y después el $19.5 \%$ ).

Impacto del DR-CAFTA en las exportaciones hacia los miembros del acuerdo comercial

En lo que respecta a las estimaciones del impacto del DR-CAFTA en las exportaciones hacia cada uno de los miembros de este acuerdo, se encontraron los siguientes resultados basándose en el método de mínimos cuadrados ordinarios.

De acuerdo a los test individuales en el modelo especificado de la tabla 2, se parte de que los betas individuales y el modelo en su forma conjunta son estadísticamente significativos, con un nivel de confianza del $95 \%$, manteniendo un nivel de confianza del $5 \%$. De esta manera, los coeficientes de determinación o bondad de ajuste muestran que las variables explicativas describen de forma muy buena a los niveles de exportaciones totales de Honduras a cada uno de los miembros del DR-CAFTA.

En este sentido, las exportaciones hacia los EE.UU. están determinadas inversamente por el ingreso de los EE.UU. y de forma positiva a través del índice términos de intercambio, la entrada en vigencia del DR-CAFTA, índice de volumen de exportaciones y la tasa de inflación. En este sentido, la implementación de este acuerdo comercial sumado al ritmo de crecimiento en el ingreso de los EE.UU. en términos del ingreso de Honduras ha contribuido en un $53 \%$ en el crecimiento anual de las exportaciones, comparado con un escenario sin la apertura del DR-CAFTA.

Tabla 2. Impacto del DR-CAFTA en las exportaciones totales de los miembros, 20002011
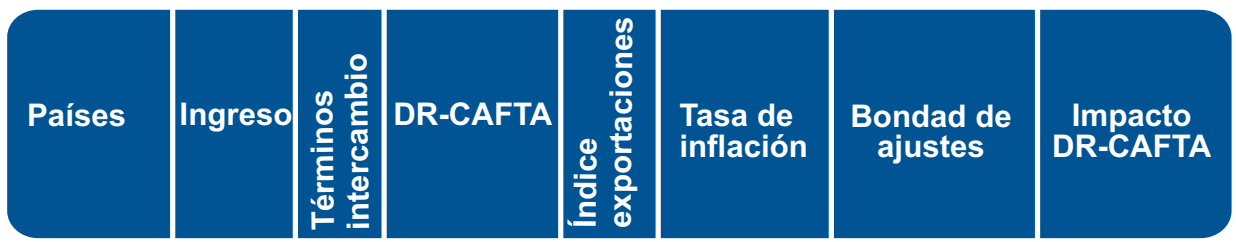

\begin{tabular}{|c|c|c|c|c|c|c|c|}
\hline EE.UU. & -0.68 & 0.72 & 0.36 & 0.35 & 0.21 & $87 \%$ & $53.0 \%$ \\
\hline Guatemala & 0.45 & 4.16 & 0.75 & 2.34 & - & $87 \%$ & $78.2 \%$ \\
\hline El Salvador & 0.43 & 3.13 & 0.56 & 2.54 & - & $86 \%$ & $64.4 \%$ \\
\hline Costa Rica & 3.71 & 4.03 & 0.82 & 3.2 & - & $79 \%$ & $83.6 \%$ \\
\hline $\mathrm{RD}$ & 2.71 & 0.75 & 1.32 & 2.96 & - & $80 \%$ & $138.5 \%$ \\
\hline Nicaragua & 2.09 & 2.2 & 1.41 & 1.32 & - & $78 \%$ & $152.1 \%$ \\
\hline
\end{tabular}

Fuente: elaboración propia con datos del BCH y Banco Mundial. 
El significativo impacto del DR-CAFTA hacia las exportaciones de los EE.UU. radica en el crecimiento del superávit de la balanza comercial agrícola, en donde a partir del periodo 2006-2011 se alcanzó un total de 4,185 millones de USD, sustentado por las exportaciones de pescados y crustáceos, moluscos y demás invertebrados acuáticos (1,110.7 millones de USD); plantas vivas y productos de la floricultura (1,422.8 millones de USD); hortalizas, plantas, raíces y tubérculos alimenticios (217.5 millones de USD); frutas y frutos comestibles, cortezas de agrios (cítricos), melones o sandías (2,243.3 millones de USD); café, té, yerba mate y especias (612.5 millones de USD). En contraste con un déficit comercial agrícola de 1,936.5 millones de USD en los restantes once capítulos de exportación agrícola.

Por su parte, la balanza comercial agrícola con Guatemala muestra un superávit de 296.3 millones de USD, explicado por las exportaciones de pescados y crustáceos, moluscos y demás invertebrados acuáticos (6.7 millones de USD); leche y productos lácteos, huevos de ave, miel natural, productos comestibles de origen animal (30.6 millones de USD); frutas y frutos comestibles, cortezas de agrios (cítricos), melones o sandías (14.5 millones de USD); café, té, yerba mate y especias (187.1 millones de USD); grasas y aceites animales o vegetales, productos de su desdoblamiento, grasas alimenticias elaboradas, ceras de origen animal o vegetal (57.1 millones de USD). También muestra un déficit comercial de 137 millones de USD, explicado principalmente por las exportaciones de productos de la molinería, malta, almidón y fécula, inulina, gluten de trigo (63.9 millones de USD) y las preparaciones de carne, pescado o de crustáceos, moluscos o demás invertebrados acuáticos (43.7 millones de USD).

El impacto generado por el DR-CAFTA hacia las exportaciones de El Salvador muestra un superávit de 456.6 millones de USD en doce capítulos de exportación agrícola, a excepción de animales vivos, cereales, productos de la molinería, malta, almidón y fécula, inulina, gluten de trigo; preparaciones de carne, pescado o de crustáceos, moluscos o demás invertebrados acuáticos con un déficit total de 165 millones de USD. De esta manera, el impacto del DR-CAFTA en las exportaciones agrícolas hacia Costa Rica recae en el superávit comercial agrícola en donde se alcanzó un total de 23.3 millones de USD en cuatro capítulos de exportación y un déficit de 153 millones de USD en el resto de exportaciones agrícolas. Antes de la entrada en vigencia de este acuerdo, el déficit comercial en el total de las exportaciones crecía a un ritmo del $21.1 \%$ y después de la apertura en un $3.9 \%$.

Por otra parte, el impacto del DR-CAFTA en las exportaciones agrícolas hacia República Dominicana recae en el superávit comercial agrícola, en donde se alcanzó un total de 9.9 millones de USD y un déficit de 6.9 millones de USD. En este 
último se muestra un nicho de mercado para las exportaciones agrícolas en seis capítulos de exportación en donde no existió intercambio comercial durante la vigencia del DR-CAFTA. De igual forma, el impacto del DR-CAFTA en las exportaciones agrícolas hacia Nicaragua recae en el superávit comercial agrícola, en donde se alcanzó un total de 28.1 millones de USD en cuatro capítulos de exportación y un déficit de 265.1 millones de USD. Este comportamiento se explica por la disminución en el déficit comercial total en donde para el periodo 2000-2005 se mostró un ritmo de crecimiento del $331 \%$ y $173 \%$ después de la entrada en vigencia del acuerdo.

Impacto del DR-CAFTA en las exportaciones agrícolas en el marco del acuerdo comercial

Las exportaciones agrícolas en promedio han mostrado una participación del 64 \% durante el periodo 2000-2011, acompañado de un crecimiento significativo del 12.7 $\%$, mayor en $2.8 \%$ que el crecimiento mostrado por el resto de bienes exportados, destacando que el año que mayor repunte obtuvo fue el 2011, con un $49.2 \%$ de crecimiento, superior en un $31.9 \%$ (883.2 millones de USD) al crecimiento mostrado en el año anterior; dicho comportamiento se sustenta por el dinamismo y competitividad en el café, banano, melones, sandías, camarón de extracción, aceite de palma, legumbres y hortalizas.

De acuerdo a los test individuales en el modelo especificado de la tabla 3, se parte de que los betas individuales y el modelo en su forma conjunta son estadísticamente significativos con un nivel de confianza del $95 \%$, manteniendo un nivel de confianza del $5 \%$. De igual forma, los coeficientes de determinación o bondad de ajuste muestran que las variables independientes explican muy bien los niveles de exportaciones agrícolas de Honduras en el marco del DR-CAFTA.

Tabla 3. Impacto de la entrada en vigencia del DR-CAFTA en las exportaciones agrícolas, 2000-2011

\begin{tabular}{|l|c|c|c|c|c|c|}
\hline Países & Ingreso & $\begin{array}{c}\text { Términos } \\
\text { intercambio }\end{array}$ & DR-CAFTA & $\begin{array}{c}\text { Índice de } \\
\text { exportaciones }\end{array}$ & $\begin{array}{c}\text { Bondad de } \\
\text { ajustes }\end{array}$ & $\begin{array}{c}\text { Impacto } \\
\text { DR-CAFTA }\end{array}$ \\
\hline EE.UU. & -1.83 & 1.28 & 0.17 & 0.88 & 89.00 & 43.54 \\
\hline Guatemala & 2.00 & 2.83 & 0.76 & 1.06 & 70.00 & 78.49 \\
\hline El Salvador & -2.49 & 0.97 & 0.19 & 0.83 & 89.00 & 44.36 \\
\hline Costa Rica & 2.72 & 0.70 & 0.54 & 1.08 & 75.00 & 62.92 \\
\hline RD & -0.24 & 2.60 & 0.79 & 1.14 & 68.00 & 80.87 \\
\hline Nicaragua & -3.20 & 1.91 & 0.33 & 0.58 & 81.00 & 51.12 \\
\hline
\end{tabular}

Fuente: elaboración propia con datos del $\mathrm{BCH}$ y Banco Mundial. 
De acuerdo a los modelos estimados se encontraron coeficientes de determinación altamente significativos, en donde puede interpretarse como el porcentaje de variabilidad de las "Xijt" explicadas por ingreso de cada uno de sus miembros del acuerdo, entrada y vigencia del DR-CAFTA, índice de volumen de exportación y la tasa de inflación. De esta forma, la entrada en vigencia del DR-CAFTA ha venido a impactar de forma positiva en el dinamismo de las exportaciones del sector agrícola en lo que respecta a los miembros del acuerdo. El mayor impacto del DR-CAFTA recae en las exportaciones agrícolas hacia República Dominicana, en donde ha contribuido con un $80.8 \%$ en el crecimiento anual de las exportaciones, comparado con un escenario sin la apertura del acuerdo; en un segundo lugar se encuentra Guatemala, con una contribución del $78.4 \%$; en un tercer lugar Costa Rica, con $62.9 \%$; en cuarto lugar Nicaragua, con $51.1 \%$; en quinto lugar El Salvador, con $44.3 \%$ y en último lugar los EE.UU. con $43.5 \%$.

El comportamiento antes mencionado radica en los crecimientos promedios en el total de las exportaciones mostrados durante el periodo 2006-2011, en donde República Dominicana mostró una participación promedio del $1.4 \%$ y un crecimiento del $40.2 \%$; seguido por Costa Rica con una participación del $4.1 \%$ y un crecimiento del $33.4 \%$; en un tercer lugar Nicaragua con el $7.3 \%$ y un crecimiento del $19.5 \%$; en un cuarto lugar Guatemala con el $11.3 \%$ y un crecimiento del $13.3 \%$; en un quinto lugar los EE.UU. con $61.8 \%$ y un crecimiento del $13.5 \%$ y por último El Salvador con $14 \%$ y un crecimiento del $13.1 \%$.

\section{Contribución delDR-CAFTA en el crecimiento económico de Honduras}

La economía hondureña, durante el periodo 2012, se caracterizó por mostrar un repunte en el crecimiento económico al registrar una tasa de $3.3 \%$ en términos reales, resultado del dinamismo experimentado en las actividades agropecuarias y manufactureras como respuesta a una mayor demanda interna y externa. Asimismo, se destaca el comportamiento positivo de las actividades de intermediación financiera, comercio, transporte, almacenamiento y comunicaciones (BCH, 2012).

Desde el punto de vista de la demanda externa, durante el 2012 se presentaron síntomas de mejoría en un $2.8 \%$, producto de los mayores niveles de exportación de café, banano, camarón congelado y empacado, azúcar y aceite de palma; en contraste, las exportaciones de prendas de vestir y productos textiles confeccionados por la industria maquiladora mostraron una desaceleración del 3.3 $\%$, influenciado por las menores niveles de importación desde el mercado norteamericano (BCH, 2012). 
En lo que respecta al impacto del DR-CAFTA en el crecimiento económico de Honduras a raíz de la entrada en vigencia del acuerdo comercial durante el periodo 2006-2011, se encontró la siguiente estimación econométrica:

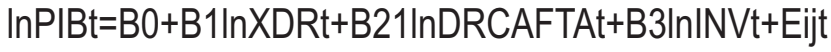

\section{En donde:}

InPIBt: corresponde al PIB a precios corrientes en USD. InXDRt: la suma de las exportaciones hacia el mercado del DR-CAFTA.

InDRCAFTAt: es una variable dicotómica en donde se le asignan valores de "0" a los años antes de entrar en vigencia el DR-CAFTA y "1" para los años a partir de la entrada en vigencia del mismo.

InINV: es la inversión directa, compuesta por la suma de la inversión directa en el exterior y la inversión directa en Honduras.

Con base a las variables previamente especificadas, el modelo de impacto del DRCAFTA en el crecimiento económico de Honduras quedaría de siguiente manera:

\section{InPIBt=14.36+0.31 lnXDRt+0.16lnDRCAFTAt+0.32InINVt+Eijt}

Tabla 4. Impacto del DR-CAFTAen el crecimiento económico, 1990-2011

- reg logpinbhn logxt DRCAFTA logind

\begin{tabular}{r|rcc} 
Source & ss & df & $\mathrm{ms}$ \\
\hline Model & 6.77292176 & 3 & 2.25764059 \\
Residual & 0.186282065 & 18 & 0.010349004 \\
\hline Total & 6.95920383 & 21 & 0.331390659
\end{tabular}

$\begin{array}{lrr}\text { Number of obs } & & 22 \\ \mathrm{f}(3, \quad 18) & = & 218.15 \\ \text { Prob }>\mathrm{F}= & 0.0000 \\ \text { R-squared }= & 0.9732 \\ \text { Adj R-squared } & = & 0.9688 \\ \text { Root MSE } & = & 0.10173\end{array}$

\begin{tabular}{c|cccccr}
\hline logpinbhn & Coef. & Std. Err. & $\mathrm{t}$ & $\mathrm{p}>|\mathrm{t}|$ & \multicolumn{2}{c}{ [95\% Conf. Interval ] } \\
\hline logxt & 0.3127374 & 0.1251817 & 2.5 & 0.022 & 0.0497404 & 0.5757344 \\
DRCAFTA & 0.1627755 & 0.0800159 & 2.03 & 0.057 & -0.0053317 & 0.3308826 \\
logind & 0.3265531 & 0.0417787 & 7.82 & 0.000 & 0.2387792 & 0.4143269 \\
_cons & 14.36694 & 2.400648 & 5.98 & 0.000 & 9.323367 & 19.41052 \\
\hline
\end{tabular}

Fuente: elaboración propia con datos del BCH y Banco Mundial.

Según el modelo estimado para el crecimiento económico se muestra un coeficiente de determinación del $96 \%$ altamente significativo, en donde puede interpretarse como el porcentaje de variabilidad de las "PIBt", explicado por el total de las exportaciones hacia el DR-CAFTA, entrada en vigencia del DR-CAFTA y el total de inversión extranjera directa. 
Con base a los resultados de la estimación econométrica, el DR-CAFTA ha impactado positivamente en el crecimiento económico de Honduras. En este sentido, la implementación de este acuerdo comercial ha contribuido en un $43.2 \%$ en el crecimiento anual del PIB, comparado con un escenario sin la apertura del DRCAFTA.

\section{DISCUSIÓN}

El dinamismo en el crecimiento de las exportaciones agrícolas es un reflejo de la sombra del crecimiento de los precios internacionales, principalmente para aquellos sectores que se caracterizan por mantener las mayores correlaciones entre los niveles de volumen de exportación y la oscilación de los precios. Este es un factor que incide significativamente en el crecimiento y participación del mercado de las exportaciones del sector agrícola en el mercado internacional, si se mantiene esta tendencia de dependencia con los precios internacionales la balanza comercial podría verse afectada en el mediano o largo plazo, en ausencia de la generación de productos agrícolas más competitivos y terminados con un valor agregado igual o mejor que el resto de economías que se fundamentan en este tipo de producción.

En este sentido, las exportaciones del sector agrícola que mayor participación poseen durante el periodo de estudio son el café, banano, camarón cultivado, aceite de palma y puros o cigarrillos; a nivel de crecimiento, los puros o cigarrillos, aceite de palma, azúcar, tilapias y cigarrillos. Las exportaciones menos competitivas son el camarón de extracción, naranjas y toronjas, cacao y sus preparaciones y las preparaciones alimenticias. En lo que respecta a las exportaciones totales, los sectores de mayor contribución son el café, banano, camarón cultivado, aceite de palma y puros o cigarrillos; por su parte, estas exportaciones se caracterizan por ser frágiles en el mercado internacional debido a que presentan elasticidades, es decir, que ante un cambio porcentual en el precio las cantidades demandadas externamente responden más que proporcionalmente a los cambios en el precio.

\section{CONCLUSIONES}

La entrada en vigencia del DR-CAFTA ha generado un impacto positivo en las exportaciones hacia cada uno de los miembros del acuerdo. En este sentido, el crecimiento anual de las exportaciones hacia los EE.UU. depende en un $53 \%$ de la implementación de este acuerdo, comparado con un escenario sin la apertura del DR-CAFTA, Guatemala $78 \%$, El Salvador $64 \%$, Costa Rica $83 \%$, Republica Dominicana $138 \%$ y Nicaragua con el $152 \%$. 
En términos de exportaciones agrícolas, la economía hondureña cuenta con un superávit comercial en el marco del DR-CAFTA, principalmente las exportaciones de frutas y frutos comestibles, cortezas de agrios (cítricos), melones o sandías; pescados y crustáceos, moluscos y demás invertebrados acuáticos; café, té, yerba mate y especias; hortalizas, plantas, raíces y tubérculos alimenticios; grasas y aceites animales o vegetales; productos de su desdoblamiento; grasas alimenticias elaboradas; ceras de origen animal o vegetal; plantas vivas y productos de la floricultura.

Al respecto, la entrada en vigencia del DR-CAFTA ha generado un impacto positivo en las exportaciones del sector agrícola, el mayor impacto del acuerdo recae en las exportaciones agrícolas hacia República Dominicana, en donde ha contribuido en un $80.8 \%$ al crecimiento anual de las exportaciones, comparado con un escenario sin la apertura del acuerdo; en un segundo lugar se encuentra Guatemala con una contribución del $78.4 \%$, en un tercer lugar Costa Rica con $62.9 \%$, cuarto lugar Nicaragua con $51.1 \%$, quinto lugar El Salvador con $44.3 \%$ y en último lugar EE.UU. con $43.5 \%$.

\section{RECOMENDACIONES}

La ejecución de los tratados de libre comercio con el resto del mundo debe manejarse de forma coordinada con los diferentes sectores productivos del país, enmarcado en brindar los mejores resultados en pro del desarrollo económico y de las políticas económicas planteadas en el Plan de Nación y Visión de País.

La economía hondureña es fuertemente dependiente del desempeño del sector agrícola, por ende, es necesaria la creación de políticas públicas encaminadas a fortalecer la productividad y el valor agregado de las exportaciones tradicionales y no tradicionales, principalmente las últimas, debido a que están sujetas a altos niveles de competitividad en el resto de países latinoamericanos.

En lo que respecta a la vigencia del DR-CAFTA, el país debe apostarle a los nichos de mercado que ofrecen los países centroamericanos y República Dominicana. Es decir, Honduras está desaprovechando oportunidades potenciales en el ámbito del sector agrícola, en donde los productores solamente están concentrados en el mercado norteamericano, olvidándose de las otras ventanas de comercio que brinda dicho tratado comercial. 


\section{AGRADECIMIENTOS}

A la Dirección de Investigación Científica y de Posgrados por aceptar y financiar mi propuesta de investigación; a mi asesor técnico, José Luis Martínez y mi asesor metodológico, Héctor Alvarado, por brindar la orientación adecuada a los resultados de esta investigación.

\section{BIBLIOGRAFÍA}

Álvarez, I.; Fischer, B. y Natera, J. (2013). MERCOSUR: Tendencias de internacionalización y capacidades tecnológicas. Revista CEPAL, 109. Santiago de Chile.

Arza, V. (2011). El Mercosur como plataforma de exportación para la industria automotriz. Revista CEPAL, 103. Santiago de Chile.

$\mathrm{BCH}$. (2013). Base de datos producto interno bruto. Recuperado de: http://www.bch.hn/cuentas_nacionales_anuales.php

$\mathrm{BCH}$. (2013). Base de datos índice de precios al consumidor. Recuperado de: http://www.bch.hn/cuentas_nacionales_anuales.php

$\mathrm{BCH}$. (2013). Base de datos exportaciones mercancías generales. Recuperado de: http://www.bch.hn/cuentas_nacionales_anuales.php

CEPAL. (2013). MagicPlus. Recuperado de: http://www.cepal.org/magic/demo.aspx?id=0

Cuevas, V. (2010). México: Dinámica de las exportaciones manufactureras. Revista CEPAL, 102. Santiago de Chile.

Timbergen, J. (1962). Shaping the World Economy: Sugestions for an International Economic Policy. Twentieth Centuty Fund, New York. Retrieved from: http://hdl.handle.net/1765/16826

Secretaría Industria y Comercio. (2011). Honduras: resultados del tratado de libre comercio entre República Dominicana, Centroamérica y Estados Unidos.

Recuperado de:

http://www.sice.oas.org/TPD/USA_CAFTA/USA_CAFTA_s.ASP

USAID. (2013). Evaluación del impacto del DR-CAFTA en los sectores productivos de República Dominicana. Recuperado de:http://seic.gov.do 